This item was submitted to Loughborough's Research Repository by the author.

Items in Figshare are protected by copyright, with all rights reserved, unless otherwise indicated.

\title{
Reduction of systematic errors in structured light metrology at discontinuities in surface reflectivity
}

PLEASE CITE THE PUBLISHED VERSION

https://doi.org/10.1016/j.optlaseng.2018.08.002

PUBLISHER

(c) Elsevier

VERSION

AM (Accepted Manuscript)

\section{PUBLISHER STATEMENT}

This paper was accepted for publication in the journal Optics and Lasers in Engineering and the definitive published version is available at https://doi.org/10.1016/j.optlaseng.2018.08.002.

LICENCE

CC BY-NC-ND 4.0

\section{REPOSITORY RECORD}

Yue, Huimin, Harshana G. Dantanarayana, Yuxiang Wu, and Jonathan Huntley. 2019. "Reduction of Systematic Errors in Structured Light Metrology at Discontinuities in Surface Reflectivity". figshare. https://hdl.handle.net/2134/34323. 


\title{
Reduction of systematic errors in structured light metrology at discontinuities in surface reflectivity
}

\author{
Huimin Yue ${ }^{1}$, Harshana G. Dantanarayana ${ }^{2}$, Yuxiang $\mathrm{Wu}^{3}$ and Jonathan M. Huntley ${ }^{2, *}$ \\ ${ }^{1}$ University of Electronic Science and Technology of China (UESTC), \\ School of Optoelectronic Science and Engineering , Chengdu 610054, China \\ ${ }^{2}$ Loughborough University, Wolfson School of Mechanical, Electrical and Manufacturing \\ Engineering, Loughborough, Leicestershire LE11 3TU, UK \\ ${ }^{3}$ Xidian University, School of Physics and Optoelectronic Engineering, Xi'an, Shaanxi, \\ 710071, China
}

*Corresponding author: J.M.Huntley@lboro.ac.uk

\begin{abstract}
In measuring 3D shape with structured light techniques, systematic errors arise in the neighbourhood of discontinuities in reflectivity or geometry. A mechanism for this phenomenon is proposed, based on the finite size of the imaging system's point spread function. A theoretical analysis for the phase errors in a phase-shifting projected fringe system is given, from which a correction algorithm to minimise the systematic errors is presented. In this algorithm, a closed form expression for the errors based on the intensity values and the phase values in a neighbourhood excluding the affected region is used to remove the estimated error from the measured phase values within the affected region. Experiments on samples with both linear and circular discontinuities in reflectivity demonstrated respective reductions in systematic errors by factors of $2.5 \times$ and $3 \times$.
\end{abstract}

Keywords: projected fringes; profilometry; edge detection; systematic errors; point spread function 


\section{Introduction}

Projected fringe profilometry has become an active research area within the field of non-contact optical full-field three-dimensional (3-D) sensing techniques because of its numerous advantages, such as fast measurement and high accuracy [1-3]. Its applications range from measuring the 3-D shape of micro-electromechanical systems (MEMS) components to the measurement of flatness of large panels. Projected Fringe Profilometry has been widely used in practical applications including object detection, digital model generation, reverse engineering, product inspection, quality control and biometric identification [4-6].

A typical fringe projection system consists of a digital projector, a digital camera and a computer. A digital camera with a matrix of pixels records the light intensity distribution of the fringe pattern that is phase modulated by the object height distribution. Quantitative information on the height distribution is normally obtained from wrapped phase maps, calculated from a series of images with different fringe phase shifts. Determination of the absolute fringe order at each pixel is typically achieved by repeating the phase measurements at a range of fringe spatial frequencies, and applying a temporal phase unwrapping algorithm [7].

Despite the relatively mature state of this technology, it is nevertheless subject to significant measurement artefacts under certain conditions. The focus of the current paper is the systematic errors that can arise when measuring surfaces with discontinuities in reflectivity by these techniques.

An example is shown in Figure 1 in which a calibration board has been measured by a commercial scanner (Phase Vision Quartz 1200 DBE). This scanner uses temporal phase shifting with a reversed exponential temporal phase unwrapping scheme [8]. The measurement is repeated at two orthogonal fringe orientations to provide additional information to improve the calibration accuracy. Figure 1(a) shows an intensity image of the calibration board. This has white circles on a black plane, a configuration that is widely used 
in projected fringe profilometry. Although the surface profile of the board is smoothly varying across the circle edges, the measured profile contains large errors in their vicinity. The height map corresponding to Fig. 1(a) is shown as a greyscale image of the full board in Fig. 1(b), a horizontal cross section in Fig. 1(c) and a mesh plot of the region around a pair of the circles in Fig. 1(d). Errors of up to $\pm 1 \mathrm{~mm}$ are clearly visible which appear to be induced by the sudden transition in reflectivity, and which will therefore be denoted 'discontinuity-induced measurement artefacts' (abbreviated to DMA) throughout the current paper.

As far as we are aware, this phenomenon has received almost no attention in the fringe projection literature. In 2003, Brakhage et al. [9] studied the reliability of phase information in the neighbourhood of object discontinuities. The errors caused by the transition between two object levels were just deleted. Although this prevents decisions being made from erroneous data, edges or corners of a sample are often the regions where data is most badly needed. A means to estimate and then to remove the errors, instead of simply discarding the data, would therefore be beneficial.

In the current paper, we give a simple physical explanation for the phenomenon, from which an analytical model to predict the spatial distribution of DMAs in the case of discontinuities in reflectivity is proposed. An algorithm is then developed that allows the correction - rather than simply deletion - of the data in the neighbourhood of a discontinuity. The performance of the correction algorithm is assessed using near-planar samples with linear and circular discontinuities in reflectivity.

\section{Model for Discontinuity-Induced Measurement Artefacts}

The principle of the fringe projection technique based on temporal phase unwrapping, and the camera/projector pinhole calibration model, is summarised in Section 2.1. Then our hypothesis for the cause of DMAs is presented in Section 2.2, from which a new analytical model is developed in Section 2.3. 


\subsection{Principle of fringe projection technique}

Temporal phase unwrapping is a method of analysing fringe patterns which is widely used in the fringe projection technique $[7,8]$. The fringe phase at each pixel is measured by standard temporal phase shifting techniques and unwrapped as a function of time. The reversed exponential temporal phase unwrapping method is used here in which the phase decreases from its maximum value to zero, with an exponentially growing series of phase changes. This provides significant improvements in reliability, accuracy, and computation time compared with the original temporal unwrapping algorithm [8].

In this paper, the fringes are projected in two sequences where the second is rotated by $90^{\circ}$ with respect to the first direction. By recording phase images with both vertical and horizontal fringe patterns, concepts from the photogrammetry literature (notably camera/projector models and nonlinear optimization techniques to estimate the system parameters) can be used to assist in the calibration [10]. At a given pixel, the unwrapped phase values for the vertical and horizontal fringes, normalised such that they each span the range $-\pi$ to $+\pi$, are represented by $\omega_{x}$ and $\omega_{y}$, respectively (see Fig. 2). These define the coordinates of a point in the image plane of the projector's spatial light modulator (SLM) through which the light illuminating scattering point $P$ must have passed. The position of $P$ is calculated by projecting rays from the camera and projector image planes through the previously-estimated lens pinhole locations $\mathbf{O}_{\mathrm{c}}$ and $\mathbf{O}_{\mathrm{p}}$. The coordinates $(X, Y, Z)$ of $P$ are defined as the midpoint of the closest points of approach of the two rays $[11,12]$.

\subsection{Proposed mechanism for DMA formation}

The cause of the artefacts is believed to be the spatial averaging of the phase signal produced by the finite size of the camera's point spread function (PSF). When a significant change in reflected light intensity occurs within the PSF, the averaged phase is weighted towards the values from the high-intensity region within the PSF, thus introducing a bias in the detected phase. 
A simple 1-D example to illustrate the effect is shown schematically in Figure 3. Figure 3(a) shows a cross section through a simulated image of a uniformly-illuminated plate which has a region of low reflectivity ( $25 \%$ of that elsewhere on the plate) over the region $-D / 2<x<D / 2$, where $D=0.4$. The imaging system is assumed here to have a PSF of negligible diameter so that a given point in the image plane receives light from only a single point on the object surface. Horizontal and vertical axes represent normalised intensity $I(x)$, and position along the plate, $x$, respectively.

The plate is then illuminated with a sequence of virtual fringe patterns, one of which is shown in Fig. 3(b). If, for example, the reversed exponential sequence [8] is used to provide an independent unwrapped phase value at each camera pixel, a sequence with 8,7,6,4 fringes across the field could be projected, with four phase shifts for each fringe frequency. Figure 3(b) then represents one of these 16 patterns, with eight fringes and a phase shift of $\pi / 2$.

The unwrapped phase profile computed from these patterns, $\omega_{x}(x)$, is shown in Fig. 3(c). The continuous and red dotted lines represent $\omega_{x}(x)$ from the high-reflectivity and low-reflectivity portions of the plate, respectively. Phase is calculated from ratios of intensity difference values. Therefore, since both the numerator and denominator are scaled equally by the local surface reflectivity, spatial variations in reflectivity have no effect on the computed phase provided there is no crosstalk between neighbouring pixels. $\omega_{x}$ thus follows a straight line with no deviations across the discontinuities in reflectivity. The subscript ' $x$ ' in $\omega_{x}$ refers to phase values coming from fringe patterns oriented normal to the $x$ axis. In the full 2-D case considered later, $\omega_{y}$ will be used to refer to the unwrapped phase map from fringes projected normal to the $y$ axis.

The key assumption made so far is that the camera recording the images has a PSF of negligible diameter compared to the field of view. Consider now the situation where this is not the case, for example due to significant defocus of the camera. Point $\mathrm{P}$ in Fig. 3(c) is a point on the sample well away from the low-reflectivity region, with a true unwrapped phase value $\omega_{x}^{\left({ }^{(P)}\right.}$. The horizontal bar represents the diameter of the PSF. The signal at P now contains contributions from the range of $\omega_{x}$ values that fall within the range of the bar. 
However since the sample is uniformly reflective over the PSF region, as seen in Fig. 3(a), the contribution from the regions to the left and right of $\mathrm{P}$, where $\omega_{x}$ is respectively greater than and less than $\omega_{x}{ }^{(\mathrm{P})}$, can be expected to largely cancel out. On the other hand, point $\mathrm{Q}$, on the left hand edge of the low-reflectivity region, receives more signal from regions of the sample with $\omega_{x} \leq \omega_{x}^{(Q)}$; the measured phase can therefore be expected to be biased in the downward direction. The opposite happens at point $\mathrm{R}$ where there is a positive bias in the measured phase.

A model for the measured phase from this finite sized PSF case is shown in Fig. 3(d), where we have assumed that the measured phase is a convolution of the true phase (Fig. 3(c)), weighted by the intensity signal (Fig. 3(a)), with the camera's PSF. The measured profile across the low reflectivity region which is obtained by removal of the linear ramp and appropriate scaling, then has positive and negative artefacts next to the low-reflectivity region which are reminiscent of those shown in Fig. 3(d). The example in Fig. 3(e) is the profile that would be obtained for the case of a $45^{\circ}$ projection angle, and the artefacts are seen to be a significant fraction of the field of view.

For many optical systems, the PSF $h(x, y)$ may be modelled as a Gaussian [13]:

$$
h\left(x-x_{c}, y-y_{c}\right)=a \mathrm{e}^{-\frac{\left(x-x_{c}\right)^{2}}{2 c^{2}}} \mathrm{e}^{-\frac{\left(y-y_{c}\right)^{2}}{2 c^{2}}}
$$

where $\left(x_{c}, y_{c}\right)$ is the position of the center of the Gaussian, $c$ controls the width which is assumed here to be identical along both the $x$ and $y$ axes, and $a$ is a normalisation factor.

The PSF of a camera can be conveniently estimated by measuring the response of the camera to an image of an object containing an edge, across which there is a step change in reflectivity. A finite difference operator acting in a direction normal to the edge then results in a Gaussian function, if the PSF is Gaussian, from which $c$ can be estimated by a simple least squares fitting procedure [13]. The parameter $c$ is related to the half width at one tenth of maximum (HWTM) of the peak and HWTM can be regarded as the radius, $R_{P}$, of the PSF. Therefore, 


$$
R_{P}=\mathrm{HWTM}=\sqrt{2 \ln 10} c \approx 2.146 c
$$

\subsection{Mathematical model for DMA}

The hypothesis underlying the current paper is that the fringe imaging system produces a recovered phase equal to a weighted average of the phase values lying within the PSF footprint, where the weighting function is a product of intensity $I$ and point spread function $h$. In 2-D, the output of the model for the phase $\bar{\omega}_{j}(j=x, y)$ recovered at point $x=x_{c}, y=y_{c}$, may thus be written as the convolution integral

$$
\bar{\omega}_{j}\left(x_{c}, y_{c}\right)=\frac{\iint_{-\infty}^{\infty} \omega_{j}(x, y) \cdot I(x, y) \cdot h\left(x-x_{c}, y-y_{c}\right) \mathrm{d} x \mathrm{~d} y}{\iint_{-\infty}^{\infty} I(x, y) \cdot h\left(x-x_{c}, y-y_{c}\right) \mathrm{d} x \mathrm{~d} y}
$$

where the denominator represents a normalisation factor.

It should be noted that Eqn. (3) represents a significant approximation in the model. A more complete analysis would require computation of the weighted intensity at $x=x_{c}, y=y_{c}$, for each of the phase-shifted images; a full error propagation calculation through the particular phase-shifting formula and phase unwrapping algorithm would then be needed to quantify the deviation in computed phase from the actual phase. Such a calculation seems impractically complex; it is therefore of interest to see how well the much simpler Eqn. (3) works with experimental data.

In order to apply Eqn. (3) it is necessary to estimate both $I$ and $\omega_{j}$ in the vicinity of the discontinuity. However, in the image plane, any measurement of these quantities within a distance $R_{P}$ of the discontinuity is affected by the values on the other side of the discontinuity. This region is illustrated in Figure 4, where the pixel grid has been rotated such that the discontinuity is locally vertical, and is labelled the PSF zone as it is a strip of width twice the radius of the Point Spread Function.

Independent Taylor series expansions in $x$ and $y$ are used on each side of the discontinuity, to represent each of the $I$ and $\omega_{j}$ distributions. The values of the Taylor series coefficients are then estimated by least squares fitting polynomial surfaces to the measured quantities within the Least Squares Fitting (LSF) zones shown in Fig. 4. Eqn. (3) then becomes 


$$
\bar{\omega}_{j}\left(x_{c}, y_{c}\right)=\frac{\iint_{-\infty}^{\infty} \widehat{\omega}_{j}(x, y) \cdot \hat{I}(x, y) \cdot h\left(x-x_{c}, y-y_{c}\right) \mathrm{d} x \mathrm{~d} y}{\iint_{-\infty}^{\infty} \hat{I}(x, y) \cdot h\left(x-x_{c}, y-y_{c}\right) \mathrm{d} x \mathrm{~d} y} .
$$

In Eqn. (4), and in what follows, the symbol ' $\wedge$ ' denotes quantities calculated from the Taylor Series coefficients, the symbol ' - , denotes the output from the model, and the symbol “ , denotes experimentally measured quantities.

For a given point inside the PSF zone at which $\bar{\omega}_{j}$ needs to be estimated, a local coordinate system is set up with the $x$ axis normal to the discontinuity, and the origin chosen to lie on the discontinuity such that $y_{c}=0$. All the results in this paper were obtained by using $1^{\text {st }}$ order Taylor series for $\omega_{j}$, and zero order series for $I$, as follows:

$$
\begin{aligned}
& \widehat{\omega}_{j}^{(r)}(x, y)=p_{j 00}^{(r)}+p_{j 10}^{(r)} x+p_{j 01}^{(r)} y \\
& \widehat{\omega}_{j}^{(l)}(x, y)=p_{j 00}^{(l)}+p_{j 10}^{(l)} x+p_{j 01}^{(l)} y \\
& \hat{I}^{(r)}(x, y)=q_{00}^{(r)} \\
& \hat{I}^{(l)}(x, y)=q_{00}^{(l)}
\end{aligned}
$$

In the above, superscripts $(r)$ and $(l)$ denote, respectively, regions to the right and the left of the discontinuity, and the $p$ and the $q$ coefficients result from least squares fitting the above equations to $\widetilde{\omega}_{j}$ and $\tilde{I}$.

For the Gaussian PSF of Eqn. (1), Eqn. (4) can be integrated to give the following analytical expression for the modelled phase:

$$
\bar{\omega}_{j}\left(x_{c}, y_{c}\right)=\frac{A+B}{C+D}
$$

where

$$
A=-\pi p_{j 00}^{(l)} q_{00}^{(l)} c^{2}\left(\operatorname{erf}\left(\frac{\sqrt{2} x_{c}}{2 c}\right)-1\right)
$$




$$
\begin{aligned}
& -\sqrt{2 \pi} p_{j 10}^{(l)} q_{00}^{(l)} c\left(c^{2} \mathrm{e}^{-\frac{x_{c}^{2}}{2 c^{2}}}-\sqrt{\frac{\pi}{2}} c x_{c}\left(1-\operatorname{erf}\left(\frac{\sqrt{2} x_{c}}{2 c}\right)\right)\right) \\
& -\pi p_{j 01}^{(l)} q_{00}^{(l)} c^{2} y_{c}\left(\operatorname{erf}\left(\frac{\sqrt{2} x_{c}}{2 c}\right)-1\right) \\
& B=-\pi p_{j 00}^{(r)} q_{00}^{(r)} c^{2}\left(\operatorname{erf}\left(\frac{\sqrt{2} x_{c}}{2 c}\right)-1\right) \\
& +\sqrt{2 \pi} p_{j 10}^{(r)} q_{00}^{(r)} c\left(c^{2} \mathrm{e}^{-\frac{x_{c}^{2}}{2 c^{2}}}+\sqrt{\frac{\pi}{2}} c x_{c}\left(1+\operatorname{erf}\left(\frac{\sqrt{2} x_{c}}{2 c}\right)\right)\right) \\
& +\pi p_{j 01}^{(r)} q_{00}^{(r)} c^{2} y_{c}\left(\operatorname{erf}\left(\frac{\sqrt{2} x_{c}}{2 c}\right)-1\right) \\
& D=\pi q_{00}^{(l)} c^{2}\left(\operatorname{erf}\left(\frac{\sqrt{2} x_{c}}{2 c}\right)+1\right) \\
& C q_{00}^{(r)} c^{2}\left(\operatorname{erf}\left(\frac{\sqrt{2} x_{c}}{2 c}\right)-1\right) \\
& \left.\begin{array}{c}
D=1 \\
C
\end{array}\right)
\end{aligned}
$$

\section{DMA correction algorithm}

In this section we describe an algorithm that estimates the error in the measured $\omega_{x}$ and $\omega_{y}$ at a given point due to a neighbouring edge discontinuity, from which a corrected coordinate can be computed. The purpose is two-fold - firstly to test the hypothesis outlined in the previous section (in particular the applicability of Eqn. (3)), and secondly to reduce DMAs in real-world applications of the projected fringe technique.

The correction algorithm includes the following four steps.

Step1 - find the affected pixels. Two edge detection methods based on that described in [14], which provides sub-pixel accuracy, and a standard Canny edge detection method [15], are used to estimate the locations of the discontinuities in the image. A dilation operation on the 
Canny detected edges, with a dilation radius equal to the radius $R_{P}$ of the PSF then generates a binary mask to specify the pixels that are potentially affected by the discontinuity. For each affected pixel, carry out the following steps 2 to 4 .

Step2 - define LSF zones. Find the nearest point on the discontinuity, as determined by the sub-pixel edge-detection method [14], to the chosen pixel. This point becomes the origin of a local coordinate system $(x, y)$ as shown in Fig. 4, with $x$ perpendicular, and $y$ parallel to the discontinuity. It is convenient to transform the camera image plane coordinates $\left(\xi_{c}, \eta_{c}\right)$ of the nearby pixels, as shown in Fig. 2, into the $(x, y)$ system by a suitable rotation/translation operation. The LSF zones are defined in this system as the two rectangular regions

$$
\begin{array}{lr}
R_{P}<x<2 R_{P} ;-R_{P}<y<R_{P} & \text { (right LSF zone) } \\
-2 R_{P}<x<-R_{P} ;-R_{P}<y<R_{P} & \text { (left LSF zone) }
\end{array}
$$

Step 3 - find Taylor series coefficients of LSF zones. The Taylor series coefficients $p_{j}^{(r)}$, $p_{j}^{(l)}, q^{(r)}$ and $q^{(l)}$ are estimated by fitting Eqns. (5)-(8) to the measured $\widetilde{\omega}_{j}$ and $\tilde{I}$ fields. The value of $\bar{\omega}_{x}$ and $\bar{\omega}_{y}$ at the current pixel, i.e., that predicted by the model, is then evaluated by Eqns. (9-13).

Step4 - calculate corrected coordinate. The error $\varepsilon_{\widetilde{\omega}_{j}}$ that will be present in the measured $\widetilde{\omega}_{j}$ at the current pixel, due to the neighbouring discontinuity, is estimated as follows:

$$
\varepsilon_{\widetilde{\omega}_{j}}=\bar{\omega}_{j}-\widehat{\omega}_{j}
$$

The measured $\widetilde{\omega}_{j}$ values are then corrected as

$$
\widetilde{\omega}_{j}^{\prime}=\widetilde{\omega}_{j}-\varepsilon_{\widetilde{\omega}_{j}}
$$

A corrected position vector for the scattering point, $\left(X^{\prime}, Y^{\prime}, Z^{\prime}\right)$, is then computed from the $\left(\widetilde{\omega}_{x}{ }^{\prime}, \widetilde{\omega}_{y}{ }^{\prime}\right)$ values from Eqns. (14) and (15), using the model for the scanner that provided the original position vector. 


\section{Experimental results}

Two objects were measured with the Phase Vision scanner to assess the effectiveness of the proposed mathematical model. One was a piece of A4 paper with printed grey and white stripes, the other was a calibration board with white circles on a black plane. Images of printed black and white stripes were also captured by the scanner's camera over a range of positions, in order to measure the variation of $R_{P}$ within the measurement volume.

The scanner comprises a single projector (ProjectionDesign F22SX+, $32 \mathrm{~mm}$ focal length, $1400 \times 1050$ pixels, $13.7 \mu \mathrm{m}$ pixel spacing in both directions) and a single camera (VDS Vosskuhler CCD 4000, $50 \mathrm{~mm}$ focal length, $2048 \times 2048$ pixels, $7.40 \mu \mathrm{m}$ pixel spacing in both directions). The lens centres of camera and projector are separated by a baseline distance of $919 \mathrm{~mm}$. The scanner has a working distance of 1.8-2.8 m, a field of view and lateral resolution of $700 \mathrm{~mm}$ and $340 \mu \mathrm{m}$, respectively, at the centre of the measurement volume, with a depth measurement accuracy of $100 \mu \mathrm{m}$.

\subsection{Measurement of PSF radius}

A typical captured image of the black/white striped sample is shown in Fig. 5(a). As the stripes are approximately vertical, the radius of the PSF can be estimated from a horizontal cross section of the image across an edge. The degree of image blur caused by the finite size of the PSF can be estimated by applying a forward difference operator to the recorded intensity values along the line, followed by a Gauss curve fitting [13]. The first order difference image of the edge response function and the Gaussian curve fitting result are shown in Fig. 5(b) and (c). Then, the radius of the PSF can be estimated by using Eqn. (2).

This procedure was repeated with the paper sample at different distances from the scanner over the range $1.8-2.8 \mathrm{~m}$, to allow the variation of $R_{P}$ with depth $Z$ within the measurement volume to be estimated. The results are presented in Fig. 5(d). By interpolating the data shown in Fig. 5(d), an estimate of $R_{P}$ at any desired height value can be obtained.

\subsection{Straight-edge sample}

The paper sample printed with grey and white stripes is a relatively simple object, which allows for a clear demonstration of the effectiveness of the proposed correction algorithm. Figure 6(a) and (b) are the Canny edge image and the dilated Canny edge image produced 
from the texture image. Figure 6(c) shows the sub-pixel edge detection image, with an enlarged part in Fig. 6(d). The red short line in the sub-pixel image denotes the position of the sub-pixel edge, and the blue arrow denotes the direction of the gradient vector.

The calibrated height of the centre point of the sample was $87 \mathrm{~mm}$, and interpolation of the data in Fig. 5(d) shows that the appropriate value of $R_{P}$ for this dataset is 4.0 pixels. A cross section of the measurement error predicted by the model, $\varepsilon_{\widetilde{\omega}_{x}}$, calculated by Eqn. (14), is shown in Fig. 7(a) for several values of $R_{P}$ close to $R_{P}=4.0$. It is compared with the difference $\delta \omega_{x}$ between the measured $\omega_{x}$ and that estimated in the PSF zone from the Taylor series coefficients, where in general

$$
\delta \omega_{j}=\widetilde{\omega}_{j}-\widehat{\omega}_{j}^{\prime}
$$

The additional prime on $\widehat{\omega}_{j}$ indicates that the least squares fitting is done over both the left and right LSF regions so that the estimated $\omega_{j}$ inside the PSF is an interpolation rather than an extrapolation. For a close-to-planar surface, $\delta \omega_{j}$ can be regarded as an estimate of the

actual measurement error, and should be equal to $\varepsilon_{\widetilde{\omega}_{j}}$ if the model and underlying hypothesis is correct. As seen in Fig. 7(a), the agreement is indeed closest between the two curves for the case $R_{P}=4$, thus providing evidence to support the validity of the model.

This value of $R_{P}$ was then used to apply the correction algorithm described in Section 3 to the data. The benefit of the DMA correction is seen clearly both in an individual cross section (Fig. 7(b)) and 3-D height map (Fig. 7(d)), compared to the uncorrected cross section and 3-D height map (Figs. 7(b) and (c) , respectively).

The RMSE (Root Mean Square Error) of the height in the PSF region for the whole image with different $R_{P}$ values is shown in Table 1 . The error reduction is again seen to be highest for the case $R_{P}=4$. With the proposed new method, and this optimal $R_{P}$ value, these systematic errors are reduced by a factor of approximately $2.5 \times$.

\subsection{Calibration board sample}

The scanner's circle calibration board provides an example that is more representative of the types of samples that may be encountered in everyday use. As the problem is now 
2-dimensional, the correction algorithm requires a decision as to whether the Taylor series are calculated along the row direction or column direction over the PSF zone. This decision is based on the orientation of each sub-pixel edge point.

Figure 8(a) and (b) shows the results of the Canny edge detection and the subsequent dilated mask, calculated from a texture image of the calibration plate. Figure 8(c) gives the sub-pixel detected edges from one of the circles. The measured height results before and after the DMA correction are shown as grey-scale images in Figure 8(d) and (e). To illustrate the correction effect more clearly, 3-D images of the two circles indicated in (d) before correction and after correction are separately given in (f) and (g), respectively. Figure 8(h) shows a cross section through the two circles before and after correction. The DMAs introduce serious errors around the circle edge area, and the proposed method shows significant ability to reduce this problem. The RMSE of the height in the PSF region of the two circles decreased from 0.41 to $0.12 \mathrm{~mm}$ by using the proposed correction method, which represents a reduction in error by a factor of over $3 \times$.

\section{Conclusions}

Significant systematic errors in measured profile are produced by the fringe projection technique in the neighbourhood of discontinuities in sample reflectivity. These are believed to be caused by the finite size of the point spread function of the camera's imaging system, which blurs the fringe signal across the discontinuities. Although the paper has only addressed fringe projection, the effect is likely to be present in all active triangulation-based optical metrology systems.

A mathematical model has been proposed from which a correction algorithm has been developed. The key input parameter to both the model and the correction algorithm is the radius of the point spread function. The effectiveness of the algorithm has been investigated with two test objects having discontinuities in reflectivity, using an independently-measured radius of the point spread function. The measurement errors were indeed found to be minimised for this radius, compared to other nearby candidate radius values, thus supporting the hypothesis for the underlying cause of the errors and the validity of the associated model. Errors in the vicinity of the discontinuity were reduced by approximately $2.5 \times$ for the linear 
stripe sample, and over $3 \times$ for the circle sample.

\section{Acknowledgements}

The authors gratefully acknowledge financial support from the Engineering and Physical Sciences Research Council under the Light Controlled Factory project EP/K018124/1. This work was also supported by National Nature Science Foundation of China (Grant No. 61421002), and the Fundamental Research Funds for the Central Universities (Grant No. JB180505). 


\section{References}

[1] J. Salvi, S. Fernandez, T. Pribanic and X. Llado, “A state of the art in structured light patterns for surface profilometry,” Pattern Recognition 43(8) 2666-2680 (2010).

[2] S. Zhang, "Recent progresses on real-time 3D shape measurement using digital fringe projection techniques,” Optics and Lasers in Engineering 48(2) 149-158 (2010).

[3] X. Su and Q. Zhang, "Dynamic 3-D shape measurement method: a review,” Optics and Lasers in Engineering 48(2) 191-204 (2010).

[4] S. S. Gorthi and P. Rastogi, "Fringe projection techniques: whither we are?" Optics and Lasers in Engineering 48(2) 133-140 (2010).

[5] Z. Wang, D. A. Nguyen, and J. C. Barnes, "Some practical considerations in fringe projection profilometry,” Optics and Lasers in Engineering 48 (2) 218-225 (2010).

[6] H.-M. Yue, X.-Y. Su, and Y.-Z. Liu, “Fourier transform profilometry based on composite structured light pattern,” Optics \& Laser Technology 39(6) 1170-1175 (2007).

[7] J. M. Huntley and H. O. Saldner, “Temporal phase unwrapping algorithm for automated interferogram analysis,” Applied Optics 32 3047-3052 (1993).

[8] J. M. Huntley and H. O. Saldner, "Error-reduction methods for shape measurement by temporal phase unwrapping,” Journal of the Optical Society of America A 14 3188-3196 (1997).

[9] P. Brakhage, M. Heinze, G. Notni, and R. Kowarschik, "Influence of the pixel size of the camera on 3d-measurements with fringe projection,” in Optical Measurement Systems for Industrial Inspection III, W. Osten, K. Creath, and M. Kujawinska, eds., Proceedings of SPIE 5144 478-483 (2003).

[10] W. Schreiber and G. Notni, “Theory and arrangements of self-calibrating whole-body three-dimensional measurement systems using fringe projection technique,” Optical Engineering 39 159-169 (2000).

[11] T. Nguyen, “Optical measurement of shape and deformation fields on challenging surfaces”. PhD thesis, https://dspace.lboro.ac.uk/2134/10551 Loughborough University (2012).

[12] J. M. Huntley, T. Ogundana, R. L. Burguete and C. R. Coggrave, “Large-scale full-field metrology using projected fringes: some challenges and solutions,” in Optical Measurement 
Systems for Industrial Inspection V, Proceedings of SPIE $661666162 C$ (2007).

[13] F. P. Wang, “The measurement of the point spread function in optical system with CCD," JGTC Int. Journal of Gannan Teachers College, Papers (06), 17-18 (2005).

[14] T. P. Agustín, K. Karl, A. F. Miguel and S. C. Daniel, “Accurate subpixel edge location based on partial area effect”, Image and Vision Computing, 31, 72-90 (2013).

[15] J. Canny, “A computational approach to edge detection,” IEEE Transactions on Pattern Analysis and Machine Intelligence, 8(6) 679-698 (1986). 


\section{Tables}

Table 1. RMSE of the estimated height in the PSF region for the whole image with different $R_{P}$ values.

\begin{tabular}{|c|c|c|c|c|c|c|}
\hline & \multicolumn{5}{|c|}{ After correction } & $\begin{array}{c}\text { Before } \\
\text { correction }\end{array}$ \\
\hline$R_{p} /$ pixels & 2 & 3 & 4 & 5 & 6 & $/$ \\
\hline RMSE/mm & 0.126 & 0.117 & 0.098 & 0.116 & 0.200 & 0.256 \\
\hline
\end{tabular}




\section{Figures}

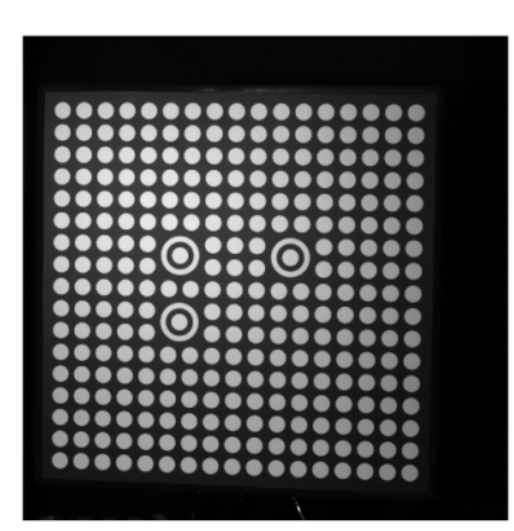

(a)

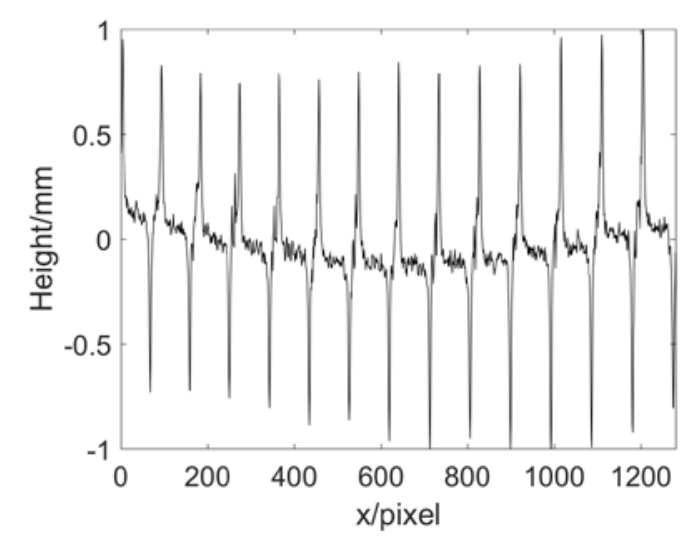

(c)

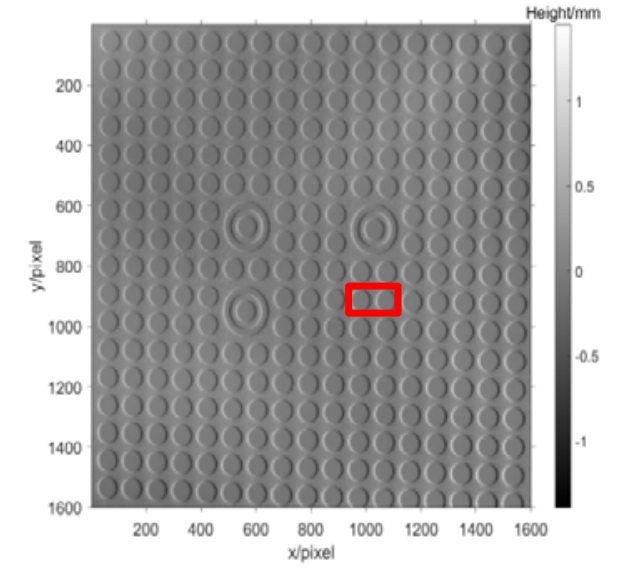

(b)

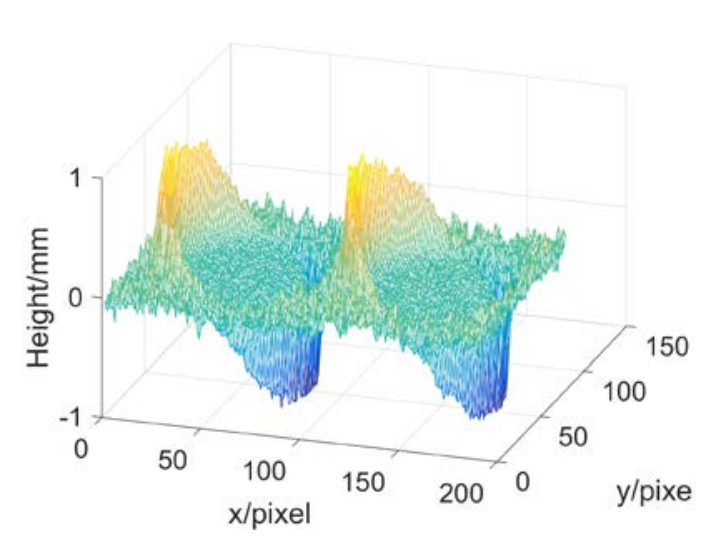

(d)

Figure 1. Calibration board with discontinuities in surface reflectivity. (a) Intensity distribution (texture image); (b) grey scale representation of height distribution after subtraction of best fit plane; (c) row 20 of (b); (d) mesh plot of the two circles in the red square indicated in (b). 


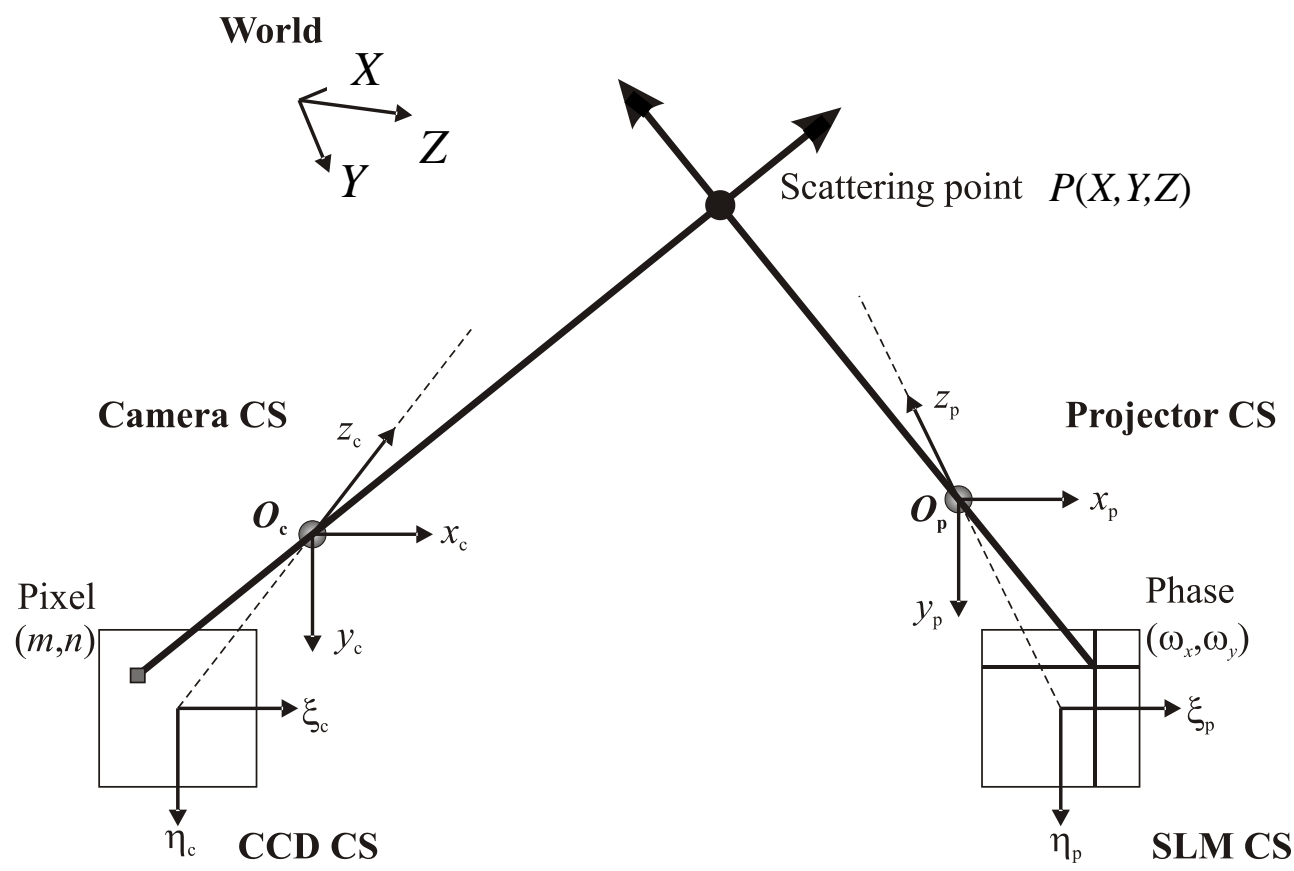

Figure 2. Pin-hole model and coordinate systems for fringe projection scanner. CCD CS $\left(\xi_{c}, \eta_{c}\right)$ is the coordinate system attached to the centre of the camera's CCD sensor. Camera CS $\left(x_{c}, y_{c}, z_{c}\right)$ is the coordinate system attached to the camera's pin-hole lens. SLM CS $\left(\xi_{p}, \eta_{p}\right)$ is the coordinate system attached to the centre of the projector's spatial light modulator (SLM). Projector CS $\left(x_{p}, y_{p}, z_{p}\right)$ is the coordinate system attached to the projector's pin-hole lens. World $\mathrm{CS}(X, Y, Z)$ is the global coordinate system in which any scattering point $P$ is defined. (Reproduced with permission from [10]). 
(a)

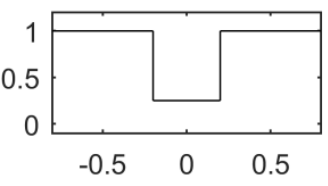

(b)

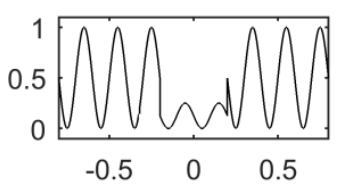

(c)

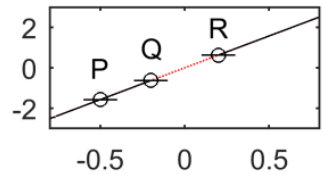

(d)

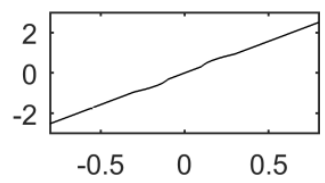

(e)

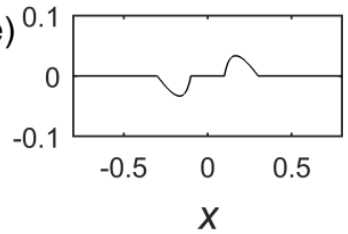

Figure 3. Schematic of DMA formation in one dimension. Image-plane intensity distribution $I(x)$ for sample containing a low-reflectivity region (25\% of the intensity of the other regions) under (a) uniform illumination and (b) fringe illumination. (c) Corresponding unwrapped phase $\omega_{x}(x)$, assuming a point spread function of zero width. (d) Modelled phase $\bar{\omega}_{x}(x)$ and (e) resultant height profile $h(x)$ for a PSF diameter of $10 \%$ of the field of view and a projection angle of $45^{\circ}$. 


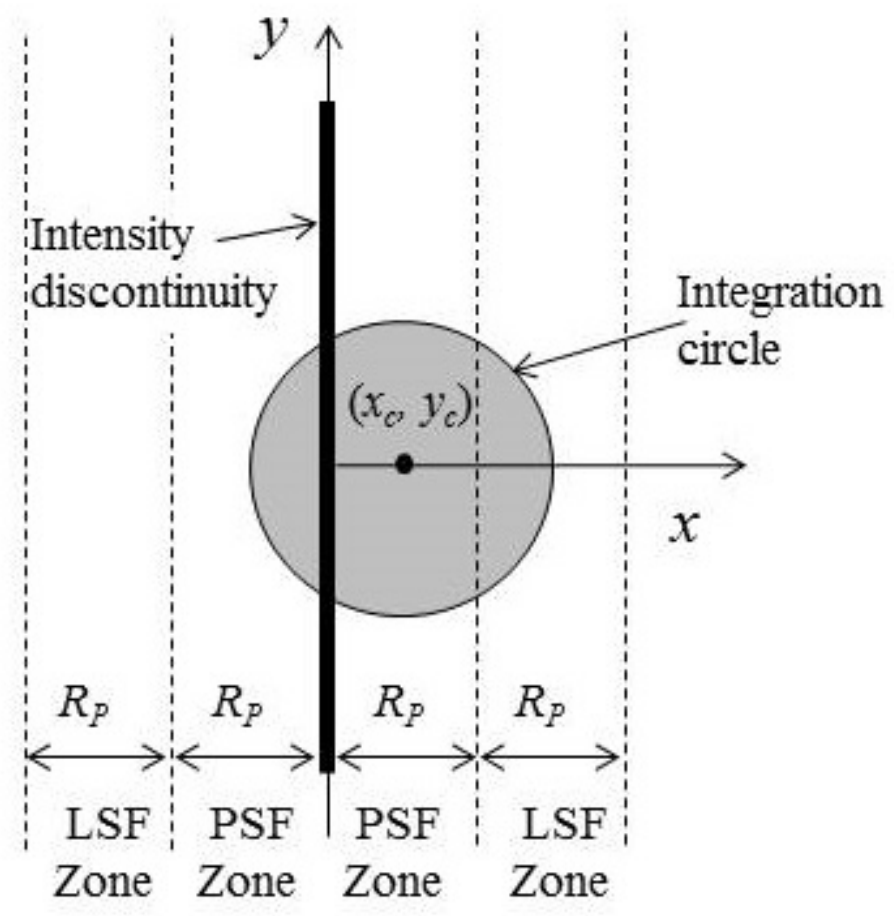

Figure 4. An image-plane point $\left(x_{c}, y_{c}\right)$ lying within distance $R_{p}$ of a discontinuity in scattered light intensity, i.e. within the 'PSF Zone', receives light from the other side of the discontinuity. 'LSF Zone' denotes the Least Squares Fitting region referred to in the text. 


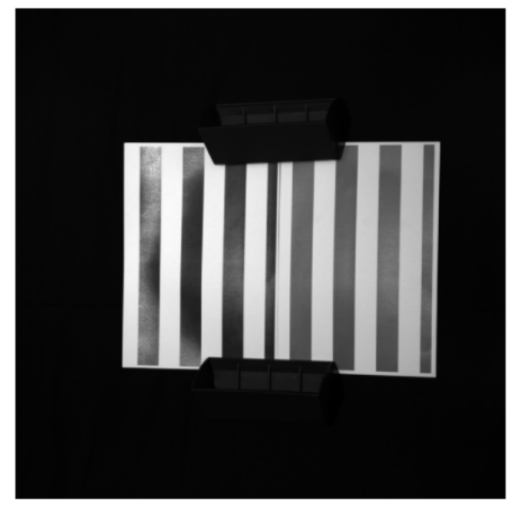

(a)

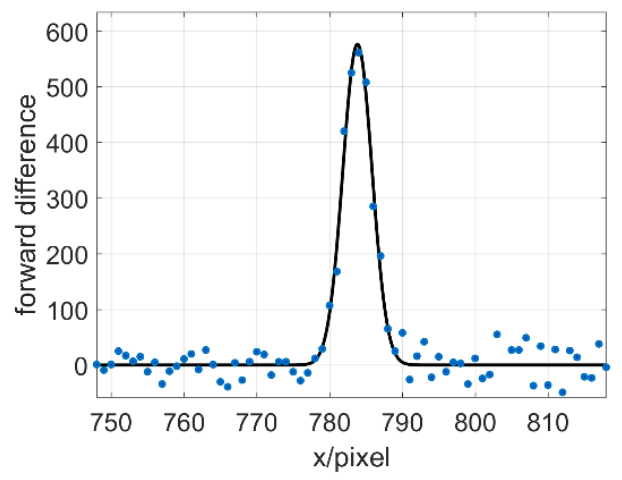

(c)

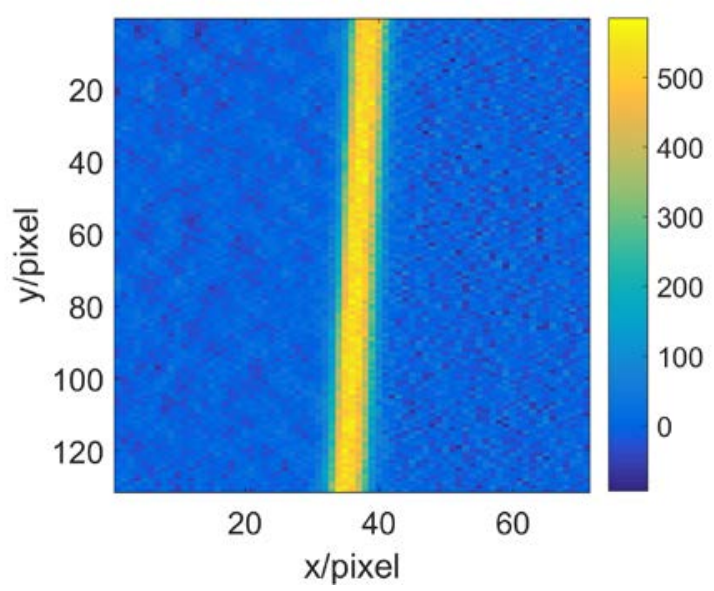

(b)

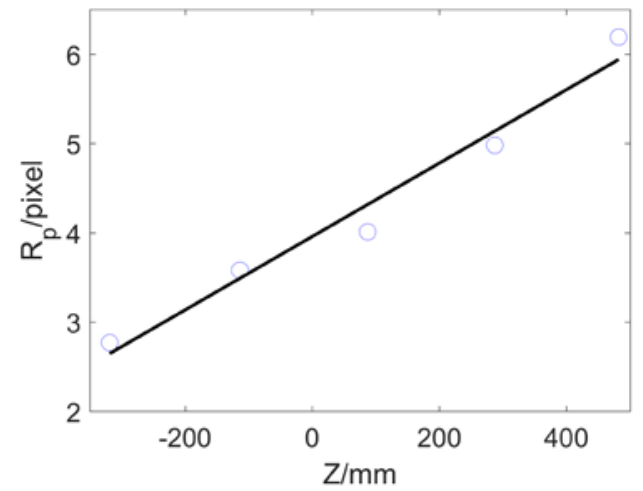

(d)

Figure 5. Measurement of PSF radius: (a) a typical captured image (the left part is the black and white stripe pattern and the right part is the grey and white stripe pattern); (b) a small region of the forward difference image near an edge; (c) Gaussian curve fitting result (continuous curve) along a line of the forward difference image (discrete points); (d) the variation of PSF radius $R_{P}$ with depth $Z$ within the measurement volume. 


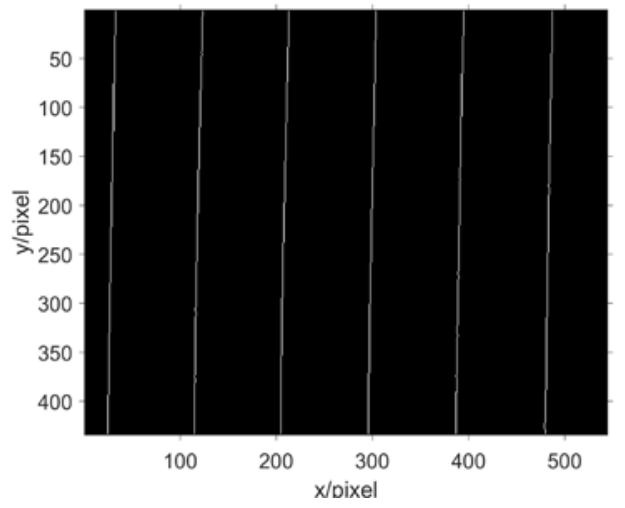

(a)

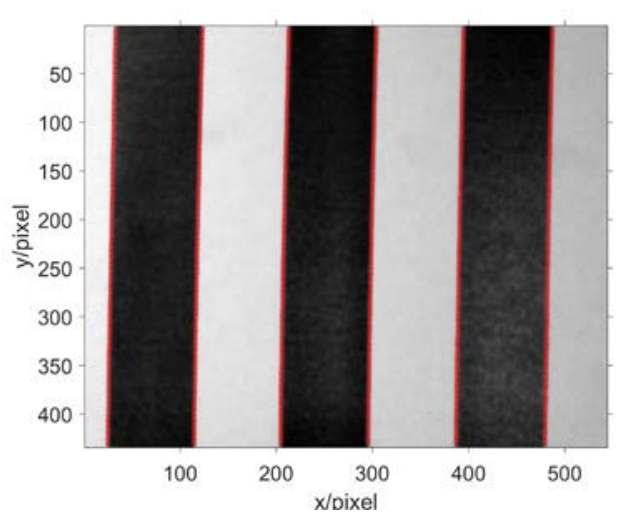

(c)

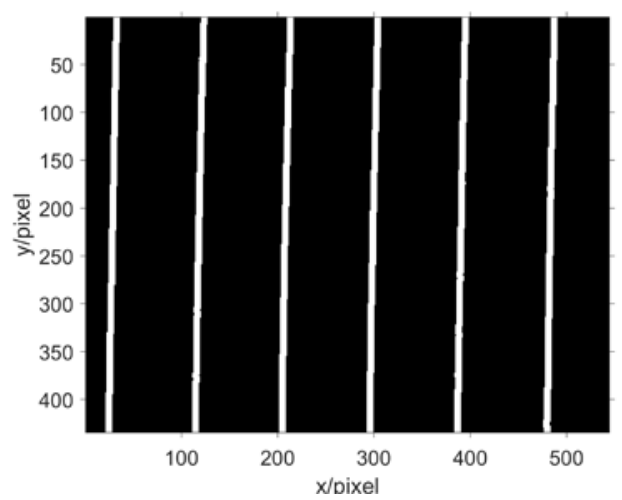

(b)

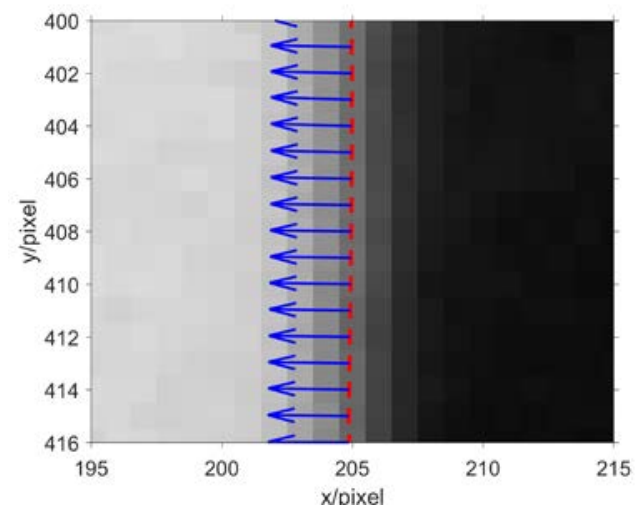

(d)

Figure 6. Edge detection on texture image from paper sample with grey and white stripes. (a) The Canny edge image; (b) the dilated Canny edge image; (c) sub-pixel image edge detection; (d) an enlarged part of (c). 


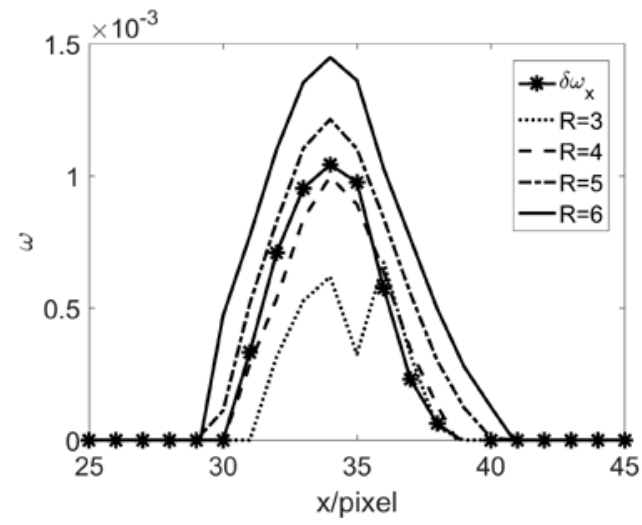

(a)

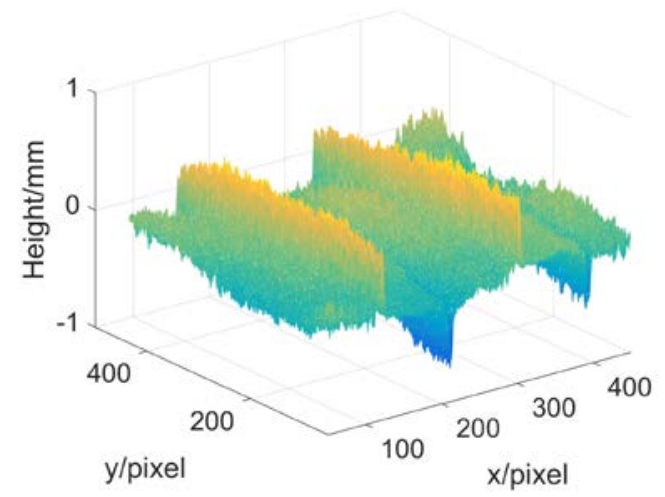

(c)

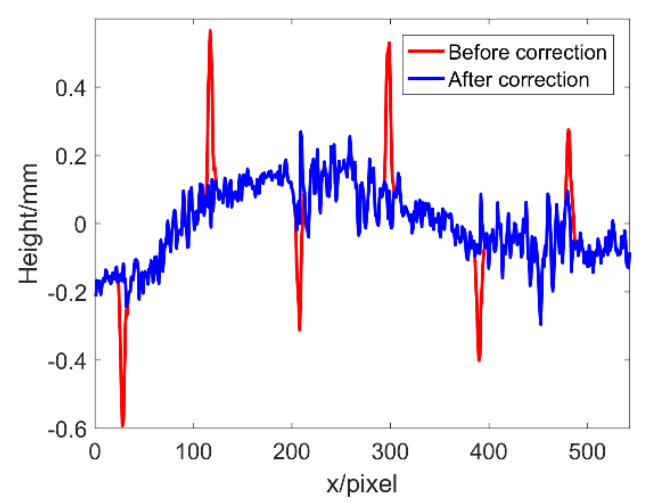

(b)

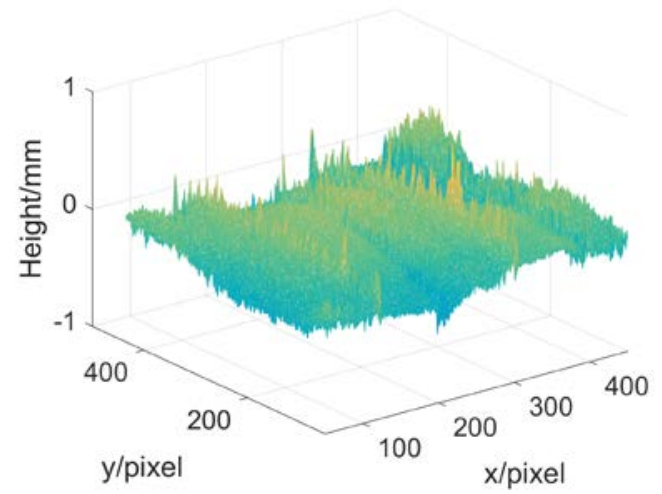

(d)

Figure 7. Measured results from paper sample with grey and white stripes. (a) $\delta \omega_{x}$ profile, and $\varepsilon_{\widetilde{\omega}_{x}}$ results with different $R_{P}$ values; (b) comparison of measured height before and after correction with proposed algorithm, using the value $R_{P}=4.0$, for one row; (c) 3-D image of height before correction; (d) 3-D image of height after correction. 


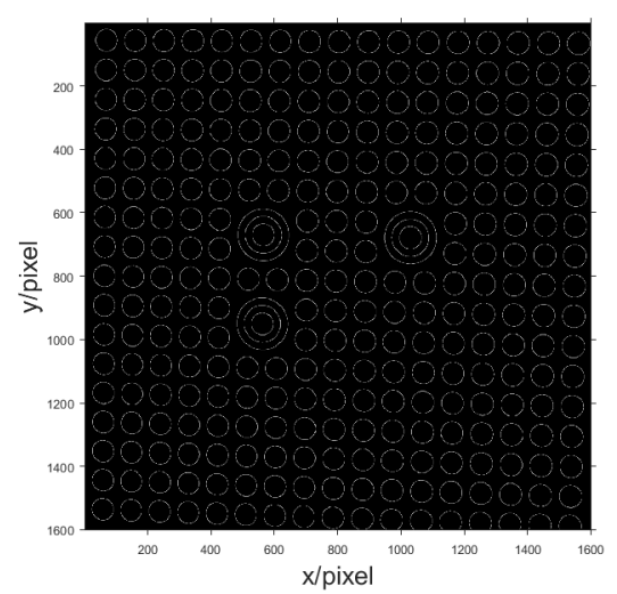

(a)

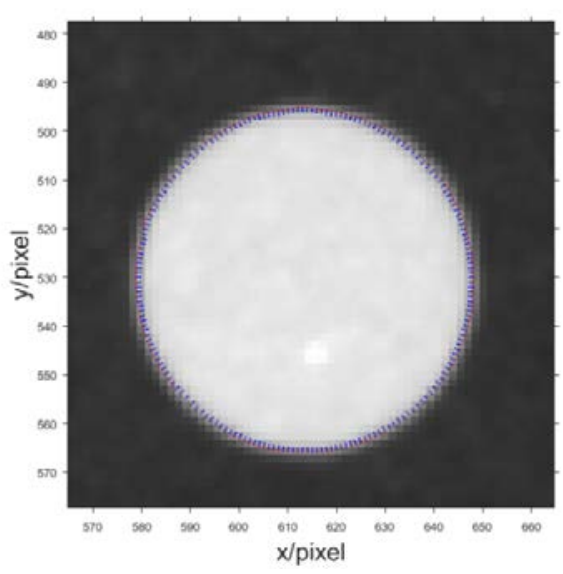

(c)

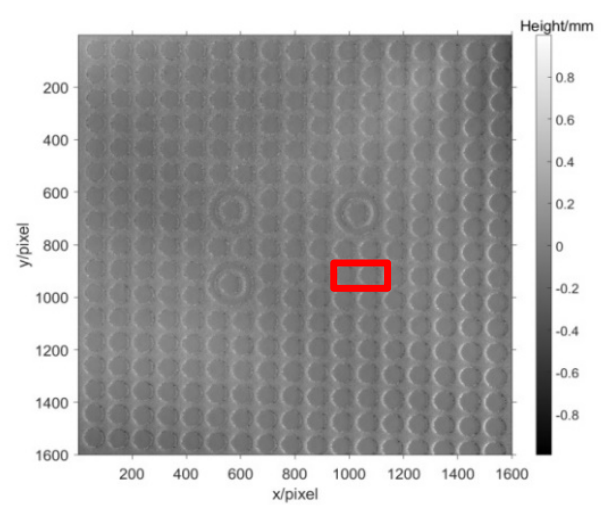

(e)

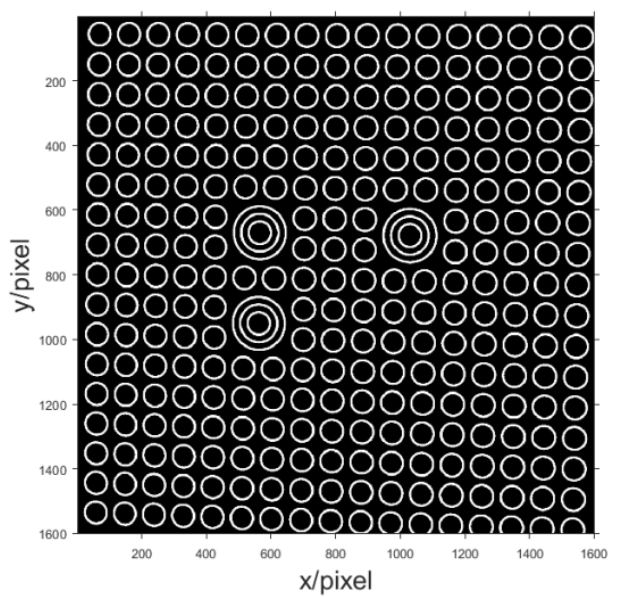

(b)

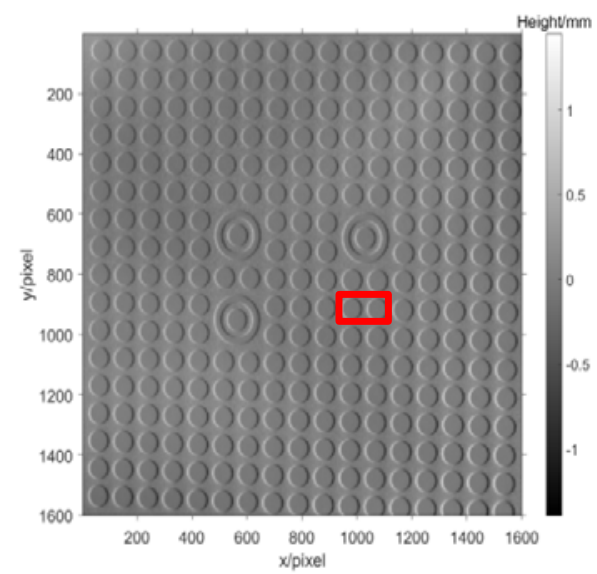

(d)

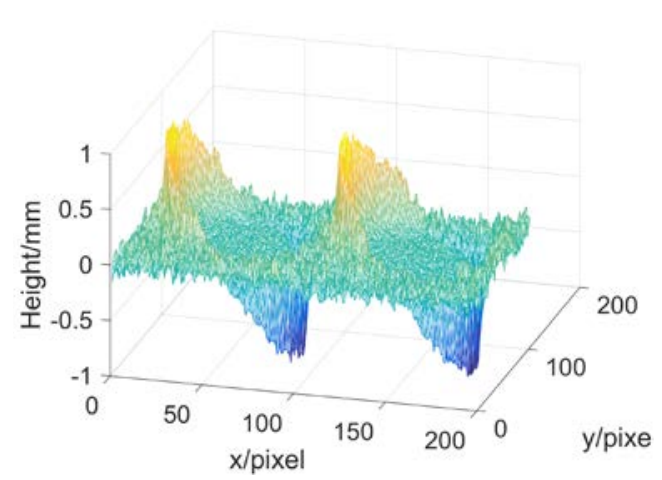

(f) 


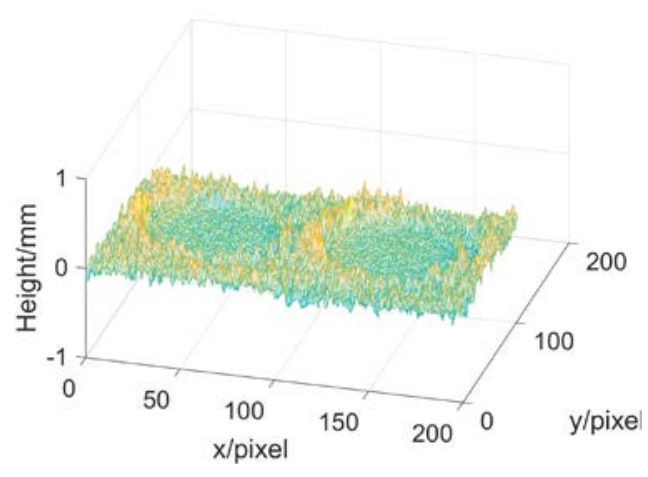

(g)

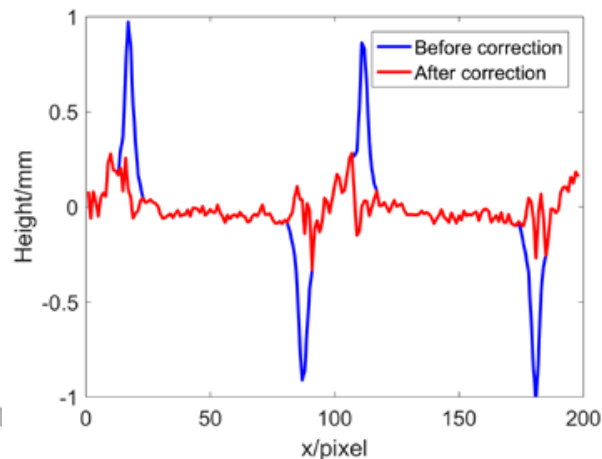

(h)

Figure 8. Measured results from circle calibration board. (a) The Canny edge image; (b) the dilated Canny edge image; (c) sub-pixel image edge detection of one circle; (d) height map of the circle artefact before correction; (e) height map of the circle artefact after correction; (f) 3-D image of the two circles indicated in (d) before correction; (g) 3D image of the two circles indicated in (e) after correction; (h) cross-section of height maps on row 50 before and after correction. 